\title{
BOSE-EINSTEIN CONDENSATION IN A RANDOM MEDIA
}

\author{
V. A. Zagrebnov \\ Université de la Méditerranée and Centre de Physique Théorique, \\ Luminy Case 90\%, 13288 Marseille, Cedex 09, France*
}

(Received January 12, 2007)

\begin{abstract}
We study the existence of condensation in a one-dimensional model of the perfect boson gas imbedded in the random Poisson potential of singular point impurities. This model manifests a type I Bose-Einstein condensation localized in a single "largest" interval of a logarithmic size (the Kac-Luttinger conjecture). It is shown that the mathematical mechanism of the Bose-Einstein condensation in this random model is identical with that in a one-dimensional nonrandom hierarchical model of scaled intervals.
\end{abstract}

Key words: neralized Bose-Einstein condensation, random potential, density of states, Lifshitz tail, Kac-Luttinger conjecture.

PACS number(s): 05.30.Jp, 03.75.Fi, 67.40.-w

\section{Contents}

I Introduction

II One-Dimensional Poisson Random Media 109

III Self-averaging of the integrated density of states

IV Thermodynamics and Bose-Einstein Condensation

V

\section{Off-Diagonal Long-Range Order}

VI

\section{Comments and discussion}

A Critical density . . . . . . . . . . 116

B Hierarchical model for BEC in onedimensional nonrandom intervals . . . . . . 117

C Statistics of large Poisson intervals . . . . . . 119

D The Kac-Luttinger conjecture . . . . . . . 120

\section{INTRODUCTION}

1.1 In our recent paper [13], we presented some general mathematical results concerning the existence of the Bose-Einstein condensation (BEC) of the Perfect BoseGas (PBG) placed in a semi-bounded from below homogeneous ergodic random external potential (random impurities). There we show that for the infinite-volume one-particle Schrödinger operator, a generic Lifshitz tail behaviour of density of states near the lower edge of the spectrum reduces the critical dimensionality of the BEC for PBG from dimensionality $d=2+\varepsilon$ to $d=1$. Therefore, the randomness enhances the BEC, and moreover, it is shown to be stable with respect to the mean-field particle interaction [12].

To tackle the corresponding Off-Diagonal Long-Range Order (ODLRO), we introduced in [13] a concept of the space-averaged one-body reduced density matrix. In spite of a rather accurate estimate of this matrix out of the BEC domain that shows an enhancement of the ODLRO exponential decay due to impurities, we did not obtain in [13] any sound estimate for this order in the BEC domain.

1.2 The aim of this paper is to present a rigorous study of a particular case of a one-dimensional PBG model in homogeneous ergodic non-negative random potential induced by the Poisson distributed singular point impurities (the Luttinger-Sy model $[17,18]$ ). We show that this model allows a rigorous mathematical approach to condensation and that one can compute explicitly some of thermodynamical quantities even in the BEC domain. This concerns in particular the ODLRO behaviour of the two-point correlation function (space-averaged one-body reduced density matrix) in the condensation regime.

Notice that the first study of possible modifications of $d=3$ dimensional BEC in the PBG caused by repulsive finite-range impurities goes back to Kac and Luttinger $[8,9]$. They predicted an enhancement of $d=3$ BEC by indication that due to impurities there is a decreasing of the critical density, but did not discuss a modification of the critical dimensionality. They also mentioned a puzzling question about the nature of the established BEC. For example, they conjectured that this condensate occurs as a macroscopic occupation of only the ground-state: type I BEC. We prove this conjecture in the case of the Luttinger-Sy model, see discussion in Section 6. We show that the nature of BEC in this model is close to what is known as the "Bose-glass", since it may be localized by the random potential. This is of interest for example in experiments with liquid ${ }^{4} \mathrm{He}$ in random environments like Aerogel and Vycor glasses, $[6,10]$.

On the other hand, the nature and behaviour of the lattice BEC may be quite different. First of all, the lattice

${ }^{*}$ UMR 6207: Université de Provence - Aix-Marseille I, Université de la Méditerranée - Aix-Marseille II, Université du Sud-Toulon-Var, FRUMAM (FR 2291),CPT-CNRS - email: zagrebnov@cpt.univ-mrs.fr 
Laplacian and the on-site Bose-Hubbard particle repulsion produces a coexistence of the BEC (superfluidity) and the Mott insulating phase as well as domains of incompressibility, see, e.g., a very complete review [23]. Adding disorder makes the corresponding models much more complicated. The physical arguments show that the randomness may suppress the BEC (superfluidity) as well as the Mott phase in favour of the localized Boseglass phase, but this is very sensitive to the choice of the random distribution, for some recent rigorous results see $[5]$.

1.3 The paper is organized as follows. In Section II we recall definition of the Luttinger-Sy model and some of its properties. We prove the self-averaging of the corresponding integrated density of states in Section III and we calculate it explicitly. In Section IV we prove that the established integrated density of states implies the existence of generalized BEC in the case of PBG.

Our main results are collected in Sections V and VI. There we recall the notion of the space-averaged onebody reduced density matrix and we prove that it has an almost sure nonrandom thermodynamic limit (selfaveraging), which can be calculated explicitly for all values of particle density. We also prove that randomness enhances decay of the two-point correlation function. In particular we show that it keeps this decay always exponential, even in the presence of BEC. We found that in the latter case the ODLRO is non-zero and that it coincides with the condensate density.

The discussion of the properties of the BEC conclude Section VI. First, we analyze the critical density dependence on the amplitude of the repulsive Poisson point impurities. Notice that for the Luttinger-Sy model the singular point impurities mean that this amplitude is infinite. Next, we study the problem of the condensate nature and its localization. To elucidate this point we invented a hierarchical one-dimensional nonrandom model, which mimics in a certain sense the (random) Luttinger-Sy model. We show that this hierarchical model can manifest different types (I, II and III) of generalized van den Berg-Lewis-Pulé condensations [1] localized in one, several or infinite number of (infinite) intervals of logarithmic sizes. To discriminate between these options, i. e. to prove or disprove the Kac-Luttinger conjecture, one has to have a fairly detailed information about the energy level spacing in random intervals generated by the Poisson impurity positions. We prove this conjecture, i. e., that type I BEC in the Luttinger-Sy model is localized in a single "largest" (i. e. infinite) interval of the logarithmic size.

\section{ONE-DIMENSIONAL POISSON RANDOM MEDIA}

In the framework of general setting this model corresponds to the following one-dimensional $(d=1)$ singleparticle random Schrödinger operator in the Hilbert space $\mathcal{H}=L^{2}(\mathbb{R})$ :

2.1 Consider a random (measurable) potential $v^{(\cdot)}(\cdot)$ :
$\Omega \times \mathbb{R} \rightarrow \mathbb{R},(\omega, x) \mapsto v^{\omega}(x)$, which is a random field on the probability space $(\Omega, \mathcal{F}, \mathbb{P})$, with the properties:

(a) $v^{\omega}$ is homogeneous and ergodic with respect to the group $\left\{\tau_{x}\right\}_{x \in \mathbb{R}}$ of probability-preserving translations on $(\Omega, \mathcal{F}, \mathbb{P})$

(b) $v^{\omega}$ is non-negative and $\inf _{x \in \mathbb{R}^{d}}\left\{v^{\omega}(x)\right\}=0$.

By $\mathbb{E}\{\cdot\}:=\int_{\Omega} \mathbb{P}(d \omega)\{\cdot\}$ we denote the expectation with respect to the probability measure in $(\Omega, \mathcal{F}, \mathbb{P})$. Then the random Schrödinger operator corresponding to the potential $v^{\omega}$ is a family of random operators $\left\{h^{\omega}\right\}_{\omega \in \Omega}$ :

$$
h^{\omega}:=t+v^{\omega},
$$

where $t:=(-\Delta / 2)$ is the free one-particle Hamiltonian, i. e., a unique self-adjoint extension of the operator: $-\Delta / 2$, with domain in $L^{2}(\mathbb{R})$.

Notice that assumptions (a) and (b) guarantee that there exists a subset $\Omega_{0} \subset \mathcal{F}$ with $\mathbb{P}\left(\Omega_{0}\right)=1$ such that operator (2.1) is essentially self-adjoint on domain $\mathcal{C}_{0}^{\infty}(\mathbb{R})$ for every $\omega \in \Omega_{0}$ (see e. g. [20] Ch.I.2).

2.2 Let $u(x) \geq 0, x \in \mathbb{R}$, be continuous function with a compact support. We call it a (repulsive) single-impurity potential. Let $\left\{\nu_{\lambda}^{\omega}(d x)\right\}_{\omega \in \Omega}$ be random Poisson measure on $\mathbb{R}$ with intensity $\lambda>0$ :

$$
\begin{aligned}
& \mathbb{P}\left(\left\{\omega \in \Omega: \nu_{\lambda}^{\omega}(\Lambda)=n\right\}\right)=\frac{(\lambda|\Lambda|)^{n}}{n !} e^{-\lambda|\Lambda|}, \\
& n \in \mathbb{N}_{0}=\mathbb{N} \cup\{0\}
\end{aligned}
$$

for any bounded Borel set $\Lambda \subset \mathbb{R}$. Then the non-negative random potential $v^{\omega}$ generated by the Poisson distributed local impurities has realizations

$$
v^{\omega}(x):=\int_{\mathbb{R}} \nu_{\lambda}^{\omega}(d y) u(x-y)=\sum_{x_{j}^{\omega} \in X^{\omega}} u\left(x-x_{j}^{\omega}\right) .
$$

Here the random set $X^{\omega}$ corresponds to impurity positions $X^{\omega}=\left\{x_{j}^{\omega}\right\}_{j} \subset \mathbb{R}$, which are the atoms of the random Poisson measure, i. e., card $\left\{X^{\omega}\lceil\Lambda\}=\nu_{\lambda}^{\omega}(\Lambda)\right.$ equals the number of impurities in the set $\Lambda$. Since the expectation is that $\mathbb{E}\left(\nu_{\lambda}^{\omega}(\Lambda)\right)=\lambda|\Lambda|$, the parameter $\lambda$ coincides with impurities concentration on the axe $\mathbb{R}$.

Remark II.1 The random potential (2.3) is obviously homogeneous and ergodic (even strongly mixing), i. e. it verifies the conditions (a) and (b). Moreover, see [20] Ch.II.5, we have that:

- There exists a nonrandom measure $d \mathcal{N}(E)$ on $\mathbb{R}$ such that

$$
d \mathcal{N}(E):=\mathbb{E}\left\{\mathcal{E}_{h^{\omega}}(d E ; 0,0)\right\}
$$

Here $\mathcal{E}_{h^{\omega}}(d E ; x, y)$ is the kernel of the spectral decomposition measure corresponding to the random Schrödinger operator $h^{\omega}$. The spectrum $\sigma\left(h^{\omega}\right)$ of $h^{\omega}$ is almost-surely (a.s.) nonrandom and it coincides with the support of $\mathcal{N}$ : $\sigma\left(h^{\omega}\right)=\operatorname{supp} \mathcal{N}$

- For repulsive impurities with compact support and for Poisson distribution, the a.s. nonrandom spectrum $\sigma\left(h^{\omega}\right)=\mathbb{R}_{+}$. Thus the lower edge of the spectrum $\inf \left\{\sigma\left(h^{\omega}\right)\right\}=0$, i. e. it coincides with the lower edge of the spectrum of the nonrandom operator $t$, see (2.1). 
- In the one-dimensional case the asymptotic behaviour of the integrated density of states $\mathcal{N}(E):=\mathcal{N}((-\infty, E])$ as $E \downarrow 0$ has the form (the Lifshitz tail):

$$
\ln \mathcal{N}(E) \sim-\lambda\left(\frac{c_{d}}{E}\right)^{d / 2}, E \downarrow 0,
$$

for $c_{d}>0$. Recall that in the nonrandom case $v^{\omega}=0$ one obtains: $\mathcal{N}(E) \sim E^{d / 2}, E \downarrow 0$.

2.3 Luttinger and Sy defined their $d=1$ model [17] restricting the single-impurity potential to the point $\delta$ potential with the amplitude $a>0$. In fact this choice (even for a more general case of random $\left\{a_{j}\right\}_{j}$ ) goes back to Frish and Lloyd [7]. Then the corresponding random potential (2.3) takes the form:

$v_{a}^{\omega}(x):=\int_{\mathbb{R}} \nu_{\lambda}^{\omega}(d y) a \delta(x-y)=a \sum_{x_{j}^{\omega} \in X^{\omega}} \delta\left(x-x_{j}^{\omega}\right)$.

Now the self-adjoint one-particle random Schrödinger operator

$$
h_{a}^{\omega}:=t \dot{+} v_{a}^{\omega},
$$

can be defined in the sense of the sum of quadratic forms. In spite of a singular nature of this random potential, by standard limiting arguments [20] it inherits the properties quoted in Remark II.1.

2.4 Moreover, the same arguments [20] are applied to define a strong resolvent (s.r.) limit of Hamiltonians (2.7), when $a \rightarrow+\infty$, which is the last step in definition of the Luttinger-Sy model, [17]. This limit gives the self-adjoint (Friedrichs) extension of the symmetric operator $t_{0}=-\Delta / 2$ with the domain $\operatorname{dom}\left(t_{0}\right)=$ $\left\{f \in \mathcal{H}: f \in \mathcal{C}_{0}^{\infty}\left(\mathbb{R} \backslash X^{\omega}\right)\right\}$. For any $\omega \in \Omega$ we denote this extension by

$$
h_{D}^{\omega}:=\text { s.r. } \lim _{a \rightarrow+\infty} h_{a}^{\omega} .
$$

Since for any $\omega \in \Omega$ the set $X^{\omega}$ can be ordered: $X^{\omega}=$ $\left\{x_{j}^{\omega}\right\}_{j}$, it generates a set of intervals $\left\{I_{j}^{\omega}:=\left(x_{j-1}^{\omega}, x_{j}^{\omega}\right)\right\}_{j}$ of lengths $\left\{L_{j}^{\omega}:=x_{j}^{\omega}-x_{j-1}^{\omega}\right\}_{j}$. Then one can decompose the Hilbert space $\mathcal{H}=L^{2}(\mathbb{R})$ into a (random) direct orthogonal sum:

$$
\mathcal{H}=\bigoplus_{j} \mathcal{H}_{j}, \quad \mathcal{H}_{j}:=L^{2}\left(I_{j}^{\omega}\right)
$$

Correspondingly, let $h_{D}\left(I_{j}^{\omega}\right)$ denote the Friedrichs extension of operator $t_{0}=-\Delta / 2$ with the domain $\operatorname{dom}\left(t_{0}\right)=$ $\left\{f \in L^{2}\left(I_{j}^{\omega}\right): f \in \mathcal{C}_{0}^{\infty}\left(I_{j}^{\omega}\right)\right\}$ :

$$
\begin{aligned}
& \left(h_{D}\left(I_{j}^{\omega}\right) f\right)(x):=-\frac{1}{2}(\Delta f)(x), \\
& f \in \operatorname{dom}\left(h_{D}\left(I_{j}^{\omega}\right)\right) \\
& =\left\{f \in W_{2}^{2}\left(I_{j}^{\omega}\right): f\left(x_{j-1}^{\omega}\right)=f\left(x_{j}^{\omega}\right)=0\right\},
\end{aligned}
$$

where $W_{2}^{2}$ denotes the corresponding Sobolev space. Then we get decompositions of the one-particle Luttinger-Sy Hamiltonian:

$$
h_{D}^{\omega}=\bigoplus_{j} h_{D}\left(I_{j}^{\omega}\right), \quad \omega \in \Omega
$$

with the domain

$$
\operatorname{dom}\left(h_{D}^{\omega}\right)=\bigoplus_{j} \operatorname{dom}\left(h_{D}\left(I_{j}^{\omega}\right)\right) \subset \mathcal{H}
$$

into random disjoint free Schrödinger operators $\left\{h_{D}\left(I_{j}^{\omega}\right)\right\}_{j, \omega}$ with Dirichlet boundary conditions at the end-points of the intervals $\left\{I_{j}^{\omega}\right\}_{j}$. The corresponding eigenfunctions have the form:

$$
\Psi_{s_{j}, D}^{\omega}(x)=\left(0,0, \ldots, \psi_{j, s_{j}}^{\omega}(x), 0, \ldots\right)
$$

with eigenvalues $\left\{E_{s_{j}}\left(L_{j}^{\omega}\right)\right\}_{s_{j}}$ :

$$
h_{D}^{\omega} \Psi_{s_{j}, D}^{\omega}=E_{s_{j}}\left(L_{j}^{\omega}\right) \Psi_{s_{j}, D}^{\omega} .
$$

Remark II.2 For a given realization $\omega \in \Omega$ the spectrum of the random operator (2.10) is explicitly defined by non-degenerate eigenvalues

$$
\sigma\left(h_{D}\left(I_{j}^{\omega}\right)\right)=\left\{E_{s_{j}}\left(L_{j}^{\omega}\right)=\frac{1}{2} \frac{\pi^{2} s_{j}^{2}}{\left(L_{j}^{\omega}\right)^{2}}\right\}_{s_{j}=1}^{\infty},
$$

with the corresponding eigenfunctions

$$
\psi_{j, s_{j}}^{\omega}(x)=\mathbb{I}_{I_{j}^{\omega}}(x) \sqrt{\frac{2}{L_{j}^{\omega}}} \sin \left(\frac{\pi s_{j}}{L_{j}^{\omega}}\left(x-x_{j-1}^{\omega}\right)\right) .
$$

Here $\mathbb{I}_{I_{j}^{\omega}}(x)$ is the characteristic function of the interval $I_{j}^{\omega}$. By consequence, the spectrum of the Luttinger-Sy Hamiltonian (2.11) is the union of (2.15)

$$
\sigma\left(h_{D}^{\omega}\right)=\bigcup_{j} \sigma\left(h_{D}\left(I_{j}^{\omega}\right)\right)
$$

By virtue of Remark II.1 this spectrum is a.s. nonrandom, and it coincides with support of the integrated density of states $\mathcal{N}$. Moreover, in the case of the Luttinger-Sy Hamiltonian (2.11) it has been known explicitly since [17]. But the rigorous study and in particular the concept of "self-averaging", which ensures this nonrandom property, are due to [16].

\section{SELF-AVERAGING OF THE INTEGRATED DENSITY OF STATES}

For the reader's convenience we recall in this section some arguments that one uses to derive the spectral properties of the Luttinger-Sy one-particle Hamiltonian. Since our aim is to study the thermodynamic properties and Bose-Einstein condensation in this model, it is useful to derive the integrated density of states first for a finite system. 
3.1 Let $\Lambda:=[-L / 2, L / 2] \subset \mathbb{R}$. Then the finite Luttinger-Sy model with the Dirichlet boundary conditions at $x= \pm L / 2$ and with $n-1$ singular pointrepulsive $(a \rightarrow+\infty)$ impurities corresponds to the onedimensional self-adjoint Schrödinger operator

$$
h_{L, X_{n}}:=\bigoplus_{j=1}^{n} h_{D}\left(I_{j}\right)
$$

acting in the direct orthogonal sum of Hilbert spaces (2.9):

$$
\mathcal{H}_{\Lambda}:=\bigoplus_{j=1}^{n} \mathcal{H}_{j}
$$

Here

$$
X_{n}=\left\{x_{0}=-L / 2<x_{1}<x_{2}<\ldots<x_{n-1}<x_{n}=L / 2\right\},\left\{I_{j}=\left(x_{j-1}, x_{j}\right)\right\}_{j=1}^{n},
$$

and operators $\left\{h_{D}\left(I_{j}\right)\right\}_{j=1}^{n}$ are defined by (2.10).

To make this system disordered, Luttinger and Sy [17] supposed that the impurity positions are random variables, which are independently and uniformly distributed over the interval $\Lambda$. Then instead of (3.3) one gets the random sets $\left\{X_{n}^{\omega}\right\}_{\omega \in \Omega}$ on $(\Omega, \mathcal{F}, \mathbb{P})$, which a.s. contain $n-1$ ordered impurities $\left\{x_{j}^{\omega}\right\}_{j=1}^{n-1}$. We denote the corresponding random Luttinger-Sy Hamiltonian and eigenfunctions in $\Lambda$ by

$$
\begin{aligned}
& h_{D, n, L}^{\omega}:=h_{L, X_{n}^{\omega}}=\bigoplus_{j=1}^{n} \\
& h_{D}\left(I_{j}^{\omega}\right), \quad h_{D, n, L}^{\omega} \Psi_{s_{j}, D, n}^{L, \omega}=E_{s_{j}}\left(L_{j}^{\omega}\right) \Psi_{s_{j}, D, n}^{L, \omega},
\end{aligned}
$$

where the eigenfunctions have the form:

$$
\Psi_{s_{j}, D, n}^{L, \omega}=\left(0,0, \ldots, \psi_{j, s_{j}}^{\omega}(x), \ldots, 0\right) \in \bigoplus_{j=1}^{n} \mathcal{H}_{j},
$$

see definitions (2.11)-(2.14).

Notice that Remark II.2 is valid in this case modulo the substitution of $h_{D}^{\omega}$ by the random operator (3.4). In particular, for the spectrum of (3.4) one gets representation:

$$
\sigma\left(h_{D, n, L}^{\omega}\right)=\bigcup_{j=1}^{n} \sigma\left(h_{D}\left(I_{j}^{\omega}\right)\right) .
$$

The following proposition is an immediate consequence of the hypothesis about the independent uniform impurities distribution and the thermodynamic limit: $L \rightarrow$ $\infty, n \rightarrow \infty$, with a fixed density of impurities

$$
\lambda=\lim _{L \rightarrow \infty} \frac{n}{L} .
$$

Proposition III.1 (a) In the thermodynamic limit the above finite-volume random point field $\left\{X_{n}^{\omega}\right\}$ converges (in distribution) to the Poisson point field $\left\{X^{\omega}\right\}$ with the intensity $\lambda$ and the corresponding random Poisson measure (2.2).

(b) The uniform and independent distribution of $n-1$ points of impurities induces on $\Lambda$ a random set of intervals $\left\{I_{j}^{\omega}\right\}_{j=1}^{n}, \omega \in \Omega$, of random lengths $\left\{L_{j}=L_{j}^{\omega}\right\}_{j=1}^{n}$. The corresponding joint probability distribution is

$$
\begin{aligned}
& d P_{L, n}\left(L_{1}, \ldots, L_{n}\right)=\frac{(n-1) !}{L^{n-1}} \\
& \times \delta\left(L_{1}+\ldots+L_{n}-L\right) d L_{1} d L_{2} \ldots d L_{n} .
\end{aligned}
$$

(c) In the thermodynamic limit the lengths $\left\{L_{j}^{\omega}\right\}_{j}$ form an infinite set of independent random variables and distribution corresponding to (3.8). It converges (weakly) to the product-measure distribution $\sigma_{\lambda}$ defined by a set of consistent marginals:

$$
d \sigma_{\lambda, k}\left(L_{j_{1}}, \ldots, L_{j_{k}}\right)=\lambda^{k} \prod_{s=1}^{k} e^{-\lambda L_{j_{s}}} d L_{j_{s}} .
$$

The proof is standard [2,22], see e.g. [12] for details.

Recall that the finite-volume integrated density of states is defined by a specific counting-function [20]. For operator (3.4), it is a random variable of the form:

$$
\begin{aligned}
\mathcal{N}_{L}^{\omega}(E): & =\frac{1}{L} \sum_{\substack{\left\{\Psi_{s, D}^{L, \omega}, n \\
s, D\right.}} \theta\left(E-E_{s, D}^{\omega}(n, L)\right) \\
& =\frac{1}{L} \int_{-L / 2}^{L / 2} d x \theta\left(E-h_{D, n, L}^{\omega}\right)(x, x) .
\end{aligned}
$$

Here $\theta\left(E-h_{D, n, L}^{\omega}\right)(x, y)$ is the kernel of the spectralprojection operator of $h_{D, n, L}^{\omega}$ corresponding to the halfline $(-\infty, E)$ and $\theta(x)=\mathbb{I}_{(0,+\infty)}(x)$ stands for the stepfunction.

Proposition III.2 In thermodynamic limit the finitevolume integrated density of states (3.10) converges a.s. to the non-random function

$$
\mathcal{N}_{\lambda}(E):=\lambda \frac{e^{-c \lambda / \sqrt{E}}}{1-e^{-c \lambda / \sqrt{E}}},
$$

with $c=\pi / \sqrt{2}$.

Proof: Explicit expressions (2.15) and (2.16) imply for (3.10) the representation:

$$
\mathcal{N}_{L}^{\omega}(E)=\frac{1}{L} \sum_{j=1}^{n} \sum_{s=1}^{\infty} \theta\left\{E-\left(\frac{c s}{L_{j}^{\omega}}\right)^{2}\right\} .
$$

Then by Proposition III.1 and by (3.7), (3.12) we obtain 


$$
\begin{aligned}
\mathcal{N}_{\lambda}(E): & =\text { a.s. } \lim _{L \rightarrow \infty} \mathcal{N}_{L}^{\omega}(E)=\text { a.s. } \lim _{n \rightarrow \infty} \frac{\lambda}{n} \sum_{j=1}^{n} \sum_{s=1}^{\infty} \theta\left\{E-\left(\frac{c s}{L_{j}^{\omega}}\right)^{2}\right\} \\
& =\lambda \mathbb{E}_{\sigma_{\lambda}}\left\{\sum_{s=1}^{\infty} \theta\left(E-\left(\frac{c s}{L_{i}}\right)^{2}\right)\right\}=\lambda^{2} \sum_{s=1}^{\infty} \int_{0}^{\infty} d L_{i} e^{-\lambda L_{i}} \theta\left(E-\left(\frac{c s}{L_{i}}\right)^{2}\right) .
\end{aligned}
$$

The a.s. limit for (non-random) integrated density of states $\mathcal{N}_{\lambda}(E)$ exists by the Birkhoff ergodic theorem $[2,22]$ and the uniform convergence of the $s$ - sum justifies the permutation of expectation with respect the $\sigma_{\lambda}$ - distribution (3.9) and the sum. Thus, we obtain:

$$
\begin{aligned}
\mathcal{N}_{\lambda}(E) & =\lambda^{2} \sum_{s=1}^{\infty} \int_{c s / \sqrt{E}}^{\infty} d L_{i} e^{-\lambda L_{i}} \\
& =\lambda \sum_{s=1}^{\infty} e^{-c s \lambda / \sqrt{E}},
\end{aligned}
$$

which yields the explicit formula (3.11).

3.2 Formula (3.11) allows us to recover for all energies $E>0$ the one-dimensional integrated density of states for the free operator $t$, i. e. the case when density of impurities $\lambda=0$, cf. Remark II.1 and (2.7):

$$
\lim _{\lambda \downarrow 0} \mathcal{N}_{\lambda}(E)=\mathcal{N}_{\lambda=0}(E)=\frac{\sqrt{2}}{\pi} \sqrt{E}
$$

Notice that for the Lebesgue-derivative $n_{\lambda}(E)$ := $d \mathcal{N}_{\lambda}(E) / d E$, i. e. for the density of states [14, Sect.4], this limit is not uniform in $E$ in the vicinity of the spectrum edge $E=0$. This confirms the argument, previously presented in [13], that the Bose-Einstein condensation in such random media cannot be viewed as a perturbation of the free case, since this phenomenon is tightly related to the behaviour of $n_{\lambda}(E)$ near the edge $[1,13]$.

On the other hand, for $\lambda>0$ and for $E$ close to the edge of the spectrum, the integrated density of states (3.11) exhibits the Lifshitz' tail behaviour:

$$
\mathcal{N}_{\lambda}(E)=\lambda e^{-c \lambda / \sqrt{E}}\left\{1-O\left(e^{-2 c \lambda / \sqrt{E}}\right)\right\}, E \downarrow 0,
$$

see Remark II.1. In this case $\lim _{E \downarrow 0} n_{\lambda}(E)=0$.

It is known $[11,16]$ that behaviour $(3.16)$ near the edge remains valid even if the parameter $a>0$ in (2.6) is $f$ nite. Notice that this parameter does not appear in the leading term of the asymptotics (3.16). This can be explained by the fact that a particle with small energy "sees" a point impurity potential with the relative amplitude of $a / E \gg 1$. Therefore, in spite of its singular nature the Luttinger-Sy Hamiltonian seems to be a good approximation for studying the BEC in Poisson random systems with non-singular repulsive impurities.

\section{THERMODYNAMICS AND BOSE-EINSTEIN CONDENSATION}

The second quantization of the one-particle LuttingerSy Hamiltonian (3.4) in the boson Fock space gives the one-dimensional PBG embedded into a random potential created by Poisson repulsive impurities (2.6) with $a=+\infty$. The latter implies that bosons are distributed over independent intervals ("boxes") $\left\{L_{j}^{\omega}\right\}_{j, \omega}$.

4.1 We study the boson Luttinger-Sy model in the grand canonical ensemble, defined by the inverse temperature $\beta>0$ and the chemical potential $\mu$. Since the model corresponds to independent "boxes" $\left\{L_{j}^{\omega}\right\}_{j, \omega}$, the grand partition function of the PBG in $\Lambda=[-L / 2, L / 2]$ is the product of partition functions calculated in individual "boxes":

$$
\begin{aligned}
\Xi_{L}^{\omega}(\beta, \mu) & =\prod_{j=1}^{n} \Xi_{L_{j}}^{\omega}(\beta, \mu) \\
& =\prod_{j=1}^{n} \prod_{s=1}^{\infty}\left(1-e^{-\beta\left(E_{s_{j}}\left(L_{j}^{\omega}\right)-\mu\right)}\right)^{-1}
\end{aligned}
$$

see (3.4). This gives for the grand canonical pressure

$$
p_{L}^{\omega}(\beta, \mu)=-\frac{1}{\beta L} \sum_{j=1}^{n} \sum_{s=1}^{\infty} \ln \left(1-e^{-\beta\left(E_{s_{j}}\left(L_{j}^{\omega}\right)-\mu\right)}\right) .
$$

To ensure convergence in (4.1) we have to bind a chemical potential from the above: $\mu<\inf _{s_{j}, \omega} E_{s_{j}}\left(L_{j}^{\omega}\right)$. By virtue of (2.15) we obtain in the thermodynamic limit:

$$
\text { a.s. } \lim _{L \rightarrow \infty} \inf _{s_{j}, \omega} E_{s_{j}}\left(L_{j}^{\omega}\right)=0 \text {. }
$$

Lemma IV.1 For $\mu<0$ and $L \rightarrow \infty$ the pressure $p_{L}^{\omega}(\beta, \mu)$ converges almost surely to the non-random function

$$
\begin{aligned}
& p(\beta, \mu)=\text { a.s. } \lim _{L \rightarrow \infty} p_{L}^{\omega}(\beta, \mu) \\
& =-\frac{1}{\beta} \int_{0}^{\infty} d E n_{\lambda}(E) \ln \left(1-e^{-\beta(E-\mu)}\right)
\end{aligned}
$$

where the limiting density of states

$$
n_{\lambda}(E):=\frac{\lambda^{2} c}{2} \frac{e^{c \lambda / \sqrt{E}}}{E^{3 / 2}\left(e^{c \lambda / \sqrt{E}}-1\right)^{2}},
$$

and $c=\pi / \sqrt{2}$, cf. (3.11). 
Proof: By the definition of integrated density of states (3.12) we can represent the pressure in (4.1) as the Lebesgue-Stieltjes integral

$$
p_{L}^{\omega}(\beta, \mu)=-\frac{1}{\beta} \int_{0}^{\infty} d \mathcal{N}_{L}^{\omega}(E) \ln \left(1-e^{-\beta(E-\mu)}\right) .
$$

Then by virtue of (3.13) we obtain that the limit

$$
\begin{aligned}
p(\beta, \mu) & =\text { a.s. } \lim _{L \rightarrow \infty} p_{L}^{\omega}(\beta, \mu) \\
& =-\frac{\lambda^{2}}{\beta} \sum_{s=1}^{\infty} \int_{0}^{\infty} d L_{i} e^{-\lambda L_{i}} \ln \left(1-e^{-\beta\left(\left(c s / L_{i}\right)^{2}-\mu\right)}\right)
\end{aligned}
$$

exists a.s. and, after a change of variables and the calculation of the sum, takes the form:

$$
\begin{aligned}
p(\beta, \mu) & =-\frac{\lambda^{2} c}{\beta} \int_{0}^{\infty} \frac{d k}{k^{2}} \frac{e^{-c \lambda / k}}{\left(1-e^{-c \lambda / k}\right)^{2}} \\
& \times \ln \left(1-e^{-\beta\left(k^{2}-\mu\right)}\right) .
\end{aligned}
$$

Setting $k=\sqrt{E}$, we recover the relation (4.3) with the density of states (4.4).

Similarly, we obtain the statement about the thermodynamic limit of the grand-canonical particle density.

Lemma IV.2 For all $\mu<0$ and $\beta>0$, the grandcanonical particles density

$$
\begin{aligned}
\rho_{L}^{\omega}(\beta, \mu) & =\frac{1}{L} \sum_{j=1}^{n} \sum_{s=1}^{\infty} \frac{1}{e^{\beta\left(E_{s_{j}}\left(L_{j}^{\omega}\right)-\mu\right)}-1} \\
& =\int_{0}^{\infty} d \mathcal{N}_{L}^{\omega}(E) \frac{1}{e^{\beta(E-\mu)}-1},
\end{aligned}
$$

converges a.s. to

$$
\rho(\beta, \mu)=\int_{0}^{\infty} d E \frac{n_{\lambda}(E)}{e^{\beta(E-\mu)}-1},
$$

with the density of states $n_{\lambda}(E)$ defined by (4.4).

Proof: By virtue of representation (4.5), the demonstration follows the same line of reasoning as we used above for the limiting pressure (4.3).
Corollary IV.1 By the explicit formula (4.4) we obtain that for the Luttinger-Sy model, defined by the Hamiltonian (2.11), the critical density

$$
\rho_{c}(\beta)=\lim _{\mu \uparrow 0} \int_{0}^{\infty} d E \frac{n_{\lambda}(E)}{e^{\beta(E-\mu)}-1}
$$

is bounded.

4.2 It is known that for PBG the condition $\rho_{c}(\beta)<\infty$ implies the existence of (generalized) Bose condensation [1], when the particles density $\rho>\rho_{c}(\beta)$. To make it obvious in our case we have to study solutions $\mu_{L}^{\omega}(\beta, \rho)$ of the finite-volume equations, see (4.5):

$$
\rho=\rho_{L}^{\omega}(\beta, \mu), \omega \in \Omega
$$

In fact, the asymptotic behaviour of $\mu_{L}^{\omega}(\beta, \rho)$ studied for a general ergodic non-negative random potential in [13]. These results then can be applied to the Luttinger-Sy model and lead to the following proposition:

Proposition IV.1 Let $\mu_{L}^{\omega}(\beta, \rho)$ be solution of equation (4.8) for the a given $\omega \in \Omega$. Then

(a) for $\rho<\rho_{c}(\beta)$ the limit

$$
\text { a.s. } \lim _{L \rightarrow \infty} \mu_{L}^{\omega}(\beta, \rho)=\mu(\beta, \rho)<0,
$$

exists and is the unique root of equation defined by (4.6):

$$
\rho=\rho(\beta, \mu)
$$

(b) for $\rho \geq \rho_{c}(\beta)$ the limit

$$
\text { a.s. } \lim _{L \rightarrow \infty} \mu_{L}^{\omega}(\beta, \rho)=0,
$$

For $\rho \geq \rho_{c}(\beta)$ the limit (4.11) implies that the density of condensed particles can be defined in the usual (for generalized condensation) way:

$$
\rho_{0}(\beta, \rho):=\lim _{\epsilon \downarrow 0}\left\{\text { a.s. } \lim _{L \rightarrow \infty} \int_{0}^{\epsilon} \mathcal{N}_{L}^{\omega}(d E) \frac{1}{e^{\beta\left(E-\mu_{L}^{\omega}(\beta, \rho)\right)}-1}\right\}=\rho-\rho_{c}(\beta),
$$

see e.g. [1]. If $\rho<\rho_{c}(\beta)$, the limit is zero. Notice that this nonrandom limit is a consequence of the chemical potential asymptotics (Proposition IV.1) and of the uniform convergence of the particle density (4.5), see [13], Theorem 4.1.

\section{OFF-DIAGONAL LONG-RANGE ORDER}

In this section we study the problem of the two-point correlation function [21,24]. By definition, in the finite volume $\Lambda$ and for any $\omega \in \Omega$, it has the form: 


\section{A. ZAGREBNOV}

$$
\begin{aligned}
\rho_{\omega}^{L}(x, y ; \beta, \mu): & =\sum_{j=1}^{n} \sum_{s_{j}=1}^{\infty} \frac{1}{e^{\beta\left(E_{s_{j}}\left(L_{j}^{\omega}\right)-\mu\right)}-1}\left(\overline{\Psi_{s_{j}, D, n}^{L, \omega}(x)}, \Psi_{s_{j}, D, n}^{L, \omega}(y)\right)_{\mathbb{C}^{n}} \\
& =\sum_{j=1}^{n} \sum_{s_{j}=1}^{\infty} \frac{1}{e^{\beta\left(E_{s_{j}}\left(L_{j}^{\omega}\right)-\mu\right)}-1} \overline{\psi_{j, s}^{\omega}}(x) \psi_{j, s}^{\omega}(y)
\end{aligned}
$$

where $(\cdot, \cdot)_{\mathbb{C}^{n}}$ denotes the scalar product in $\mathbb{C}^{n}$, see $(3.4)$ and (3.5). Therefore (5.1) is the kernel of for the one-body reduced density matrix, see e.g. [15].

5.1 We know that this function is not self-averaging in the thermodynamic limit $[16,20]$. To get a way out, we proposed in [13] to consider the space-averaged version of (5.1):

$\tilde{\rho}_{\omega}^{L}(x, y ; \beta, \mu):=\frac{1}{L} \int_{-L / 2}^{L / 2} d z \rho_{\omega}^{L}(\beta, \mu ; x+z, y+z)$

The motivation was based on the fact that in the limit $\lambda \downarrow 0$, we recover from (5.2) the free case, see [13] and subsection V.2 below.
Theorem V.1 For the Luttinger-Sy model, the thermodynamic limit of the space-averaged two-point correlation function (5.2) for $\beta>0$ and $\mu \leq 0$, is a.s. nonrandom and has the form:

$$
\tilde{\rho}(x, y ; \beta, \mu)=\rho_{0}(\beta, \rho)+e^{-\lambda|x-y|} \Phi_{\lambda}(|x-y|) .
$$

Here $\Phi_{\lambda}(|x-y|)$ is defined by (5.6). The chemical potential $\mu=\mu(\beta, \rho)$, and the condensate density $\rho_{0}(\beta, \rho)$ (4.12) are related to the total particle density $\rho$.

Proof: We consider first the case of a negative chemical potential (4.9), i. e. $\mu=\mu(\beta, \rho)<0$ and $\rho_{0}(\beta, \rho)=0$. Using the explicit form of eigenfunctions (2.16), we obtain for the thermodynamic limit of (5.2):

$$
\begin{aligned}
& \tilde{\rho}(x, y ; \beta, \mu)=\lim _{L \rightarrow \infty} \frac{\lambda}{n} \sum_{j=1}^{n} \sum_{s_{j}=1}^{\infty} \frac{1}{e^{\beta\left(E_{s_{j}}\left(L_{j}^{\omega}\right)-\mu\right)}-1} \\
& \times \frac{2}{L_{j}^{\omega}} \int_{-L / 2}^{L / 2} d z \sin \left(k_{s_{j}}\left(L_{j}^{\omega}\right)\left(x+z-x_{j-1}^{\omega}\right)\right) \sin \left(k_{s_{j}}\left(L_{j}^{\omega}\right)\left(y+z-x_{j-1}^{\omega}\right)\right) \mathbb{I}_{I_{j}^{\omega}}(x+z) \mathbb{I}_{I_{j}^{\omega}}(y+z),
\end{aligned}
$$

with $k_{s_{j}}\left(L_{j}^{\omega}\right):=\sqrt{2 E_{s_{j}}\left(L_{j}^{\omega}\right)}$. Let for simplicity $x>y$. Then the integration reduces to the interval $\left[x_{j-1}^{\omega}-y, x_{j}^{\omega}-x\right]=$ $\left[x_{j-1}^{\omega}-y, x_{j-1}^{\omega}-x+L_{j}^{\omega}\right]$, such that $(x-y) \leq L_{j}^{\omega}$. Since $k_{s_{j}}\left(L_{j}^{\omega}\right) L_{j}^{\omega}=s_{j} \pi$, the integration over $z$ yields

$$
\begin{aligned}
& \frac{2}{L_{j}^{\omega}} \int_{-L / 2}^{L / 2} d z \sin \left(k_{s_{j}}\left(L_{j}^{\omega}\right)\left(x+z-x_{j-1}^{\omega}\right)\right) \sin \left(k_{s_{j}}\left(L_{j}^{\omega}\right)\left(y+z-x_{j-1}^{\omega}\right)\right) \mathbb{I}_{I_{j}^{\omega}}(x+z) \mathbb{I}_{I_{j}^{\omega}}(y+z) \\
& =\theta\left(L_{j}^{\omega}-(x-y)\right)\left\{\cos \left(k_{s_{j}}\left(L_{j}^{\omega}\right)(x-y)\right)\left[1-(x-y) / L_{j}^{\omega}\right]+\frac{1}{s_{j} \pi} \sin \left(k_{s_{j}}\left(L_{j}^{\omega}\right)(x-y)\right)\right\},
\end{aligned}
$$

with step function $\theta(z)$. Since by Proposition III.1 the random variables $L_{j}^{\omega}$ are independent in the limit $L \rightarrow \infty$, we apply to (5.4) the Birkhoff ergodic theorem. This yields the limit:

$$
\begin{aligned}
\tilde{\rho}(x, y ; \beta, \mu) & =\text { a. s. } \lambda^{2} \sum_{s=1}^{\infty} \int_{0}^{\infty} d L e^{-\lambda L} \frac{1}{e^{\left.\beta\left(\left(c s_{j} / L\right)^{2}\right)-\mu\right)}-1} \theta(L-(x-y)) \\
& \times\left\{\cos (\sqrt{2} \operatorname{cs}(x-y) / L)[1-(x-y) / L]+\frac{1}{s \pi} \sin (\sqrt{2} \operatorname{cs}(x-y) / L)\right\} .
\end{aligned}
$$

with $c=\pi / \sqrt{2}$. If we put $q=c s / L$, then

$$
\begin{aligned}
& \tilde{\rho}(x, y ; \beta, \mu)=\lambda^{2} \sum_{s=1}^{\infty} \int_{0}^{\infty} \frac{d q}{q^{2}} e^{-c s \lambda / q} \frac{1}{e^{\beta\left(q^{2}-\mu\right)}-1} \\
& \times c \theta(s-q(x-y) / c)\left\{\cos (\sqrt{2} q(x-y))[s-q(x-y) / c]+\frac{1}{\pi} \sin (\sqrt{2} q(x-y))\right\} .
\end{aligned}
$$

Two sums over $s$ give: 


$$
\begin{aligned}
& \sum_{s=1}^{\infty} e^{-c s \lambda / q} \theta(s-q(x-y) / c)[s-q(x-y) / c]=e^{-e n t[q(x-y) / c] \lambda c / q}\left\{\frac{e^{-\lambda c / q}}{\left(1-e^{-\lambda c / q}\right)^{2}}-\frac{\operatorname{frac}\{q(x-y) / c\} e^{-\lambda c / q}}{1-e^{-\lambda c / q}}\right\} \\
& \sum_{s=1}^{\infty} e^{-c s \lambda / q} \theta(s-q(x-y) / c)=e^{-e n t[q(x-y) / c] \lambda c / q} \frac{e^{-\lambda c / q}}{1-e^{-\lambda c / q}}
\end{aligned}
$$

where ent $[u]$ and frac $\{u\}$ denote correspondingly the entire and the fractional parts of $u \in \mathbb{R}$. Since $q(x-y) / c=$ ent $[q(x-y) / c]+\operatorname{frac}\{q(x-y) / c\}$, after a change of variables, $q=\sqrt{E}$, we obtain by (3.11), (4.4) and (5.5) :

$$
\begin{aligned}
\tilde{\rho}(x, y ; \beta, \mu) & =e^{-\lambda|x-y|} \int_{0}^{\infty} d E \frac{n_{\lambda}(E)}{e^{\beta(E-\mu)}-1} e^{\varphi(|x-y|, \lambda, E)} \cos (\sqrt{2 E}(x-y)) \\
& -e^{-\lambda|x-y|} \frac{\lambda}{2} \int_{0}^{\infty} \frac{d E}{E^{3 / 2}} \frac{\mathcal{N}_{\lambda}(E)}{e^{\beta(E-\mu)}-1} e^{\varphi(|x-y|, \lambda, E)} \\
& \times c\left\{\operatorname{frac}\{\sqrt{E}(x-y) / c\} c \cos (\sqrt{2 E}(x-y))-\frac{1}{\pi} \sin (\sqrt{2 E}(x-y))\right\}=: e^{-\lambda|x-y|} \Phi_{\lambda}(|x-y|) .
\end{aligned}
$$

We put here $\varphi(|x-y|, \lambda, E):=c \lambda \operatorname{frac}\{\sqrt{E}|x-y| / c\} / \sqrt{E}$ and $|x-y|$, since the proof for $x-y \leq 0$ is identical to that for $0 \leq x-y$.

Notice that the positive function frac $\{\sqrt{E}|x-y| / c\} \in(0,1)$. Hence, for $\lambda>0$ and for any (large) $|x-y| \gg 1$ there exist $\tilde{E}(|x-y|)>0$ such that frac $\{\sqrt{E}|x-y| / c\}=\sqrt{E}|x-y| / c$ for $E<\tilde{E}(|x-y|)$, i. e., $\varphi(|x-y|, \lambda, E)=\lambda|x-y|$. By (3.11) and (4.4) the integrated density of states $\mathcal{N}_{\lambda}(E)$ and the density of states $n_{\lambda}(E)$ converge for $E \downarrow 0$ to zero faster than any power of $E$. By the Riemann-Lebesgue lemma this implies that the integrals (5.6) over interval $\left[0, E_{-}(|x-y|)\right]$, where $E_{-}(|x-y|)<\tilde{E}(|x-y|)$, can be made of the order $|x-y|^{-\alpha}$ for any (large) $\alpha>0$. On the other hand, integrals (5.6) converge on $\left[E_{-}(|x-y|), \infty\right)$ uniformly in $|x-y|$. This proves the representation (5.6) with a bounded function $\Phi_{\lambda}(|x-y|)$.

Now we study the case when the condensate exists. The finite-volume chemical potential $\mu_{L}^{\omega}(\beta, \rho)$ is a solution of equation (4.8). By Proposition IV.1(b) for $\rho>\rho_{c}(\beta)$ it implies (4.11), i. e. $\mu_{L}^{\omega}\left(\beta, \rho>\rho_{c}(\beta)\right)$ converges a. s. to 0 . To find the limit of space-averaged correlation function (5.2) for the sequence $\left\{\mu_{L}^{\omega}(\beta, \rho)\right\}_{L}$ we split (5.2) into two parts:

$$
\begin{aligned}
& \tilde{\rho}_{L}^{\omega}\left(x, y ; \beta, \mu_{L}^{\omega}(\beta, \rho)\right)=\frac{1}{L} \sum_{j=1}^{n} \sum_{s_{j}=1}^{\infty} \frac{1}{e^{\beta\left(E_{s_{j}}\left(L_{j}^{\omega}\right)-\mu_{L}^{\omega}(\beta, \rho)\right)}-1} \theta\left(\delta-E_{s_{j}}\left(L_{j}^{\omega}\right)\right) \\
& \times \theta\left(L_{j}^{\omega}-(x-y)\right)\left\{\cos \left(k_{s_{j}}\left(L_{j}^{\omega}\right)(x-y)\right)\left[1-(x-y) / L_{j}^{\omega}\right]+\frac{1}{s_{j} \pi} \sin \left(k_{s_{j}}\left(L_{j}^{\omega}\right)(x-y)\right)\right\} \\
& +\frac{1}{L} \sum_{j=1}^{n} \sum_{s_{j}=1}^{\infty} \frac{1}{e^{\beta\left(E_{s_{j}}\left(L_{j}^{\omega}\right)-\mu_{L}^{\omega}(\beta, \rho)\right)}-1} \theta\left(E_{s_{j}}\left(L_{j}^{\omega}\right)-\delta\right) \\
& \times \theta\left(L_{j}^{\omega}-(x-y)\right)\left\{\cos \left(k_{s_{j}}\left(L_{j}^{\omega}\right)(x-y)\right)\left[1-(x-y) / L_{j}^{\omega}\right]+\frac{1}{s_{j} \pi} \sin \left(k_{s_{j}}\left(L_{j}^{\omega}\right)(x-y)\right)\right\},
\end{aligned}
$$

for some $\delta>0$. Since in the second term of the right-hand side of (5.7) the eigenvalues $E_{s_{j}}\left(L_{j}^{\omega}\right) \geq \delta>0$, the limit (4.11) and uniform convergence of the sums (cf. Corollary 3.1 in [13]) yields

$$
\begin{aligned}
& \lim _{\delta \downarrow 0} \lim _{L \rightarrow \infty} \tilde{\rho}_{L}^{\omega}\left(x, y ; \beta, \mu_{L}^{\omega}(\beta, \rho)\right)=\text { a.s. } \lim _{\delta \downarrow 0} \lim _{L \rightarrow \infty} \frac{1}{L} \sum_{i=1}^{\nu} \sum_{s_{j}=1}^{\infty} \frac{1}{e^{\beta\left(E_{s_{j}}\left(L_{j}^{\omega}\right)-\mu_{L}^{\omega}(\beta, \rho)\right)}-1} \theta\left(\delta-E_{s_{j}}\left(L_{j}^{\omega}\right)\right) \\
& \times \theta\left(L_{j}^{\omega}-(x-y)\right)\left\{\cos \left(k_{s_{j}}\left(L_{j}^{\omega}\right)(x-y)\right)\left[1-(x-y) / L_{j}^{\omega}\right]+\frac{1}{s_{j} \pi} \sin \left(k_{s_{j}}\left(L_{j}^{\omega}\right)(x-y)\right)\right\}+\tilde{\rho}(x, y ; \beta, 0),
\end{aligned}
$$

where the last term is defined by (5.6).

To study the limit of the first term in the right-hand side of (5.8) we use the fact that by (2.15) the levels with energies $E_{s_{j}}\left(L_{j}^{\omega}\right)<\delta$ are all localized in large "boxes" with the length of the order of $O\left(\delta^{-1 / 2}\right)$. Then for $E_{s_{j}}\left(L_{j}^{\omega}\right)<\delta$ and $|x-y|=o\left(\delta^{-1 / 2}\right)$, with $\delta \rightarrow 0$, we obtain the asymptotics

$$
\theta\left(L_{j}^{\omega}-(x-y)\right)\left\{\cos \left(k_{s_{j}}\left(L_{j}^{\omega}\right)(x-y)\right)\left[1-(x-y) / L_{j}^{\omega}\right]+\frac{1}{s_{j} \pi} \sin \left(k_{s_{j}}\left(L_{j}^{\omega}\right)(x-y)\right)\right\}=1+\delta^{1 / 2} o\left(\delta^{-1 / 2}\right) .
$$

Therefore, by definition of generalized condensation (4.12) and by (5.8), we get

$$
\tilde{\rho}_{L}^{\omega}(x, y ; \beta, 0):=\text { a.s. } \lim _{\delta \downarrow 0} \lim _{L \rightarrow \infty} \tilde{\rho}_{L}^{\omega}\left(x, y ; \beta, \mu_{L}^{\omega}(\beta, \rho)\right)=\rho_{0}(\beta, \rho)+\tilde{\rho}(x, y ; \beta, 0),
$$

for $\rho>\rho_{c}(\beta)$. This finishes the proof of (5.3). 


\section{A. ZAGREBNOV}

5.2 It is instructive to make a contact between the concept of the space-averaged one-body reduced density matrix (5.2) and the one for nonrandom free PBG.

Corollary V.1 When density of particles $\rho$ exceeds the critical value $\rho_{c}(\beta)(4.7)$, the space-averaged one-body reduced density matrix of the Luttinger-Sy model, manifests Off-Diagonal Long-Range Order:

$$
O D L R O(\beta, \rho):=\lim _{|x-y| \rightarrow \infty} \tilde{\rho}(x, y ; \beta, \mu(\beta, \rho))=\rho_{0}(\beta, \rho),
$$

We see that similarly to the nonrandom case this limit is defined by the condensation density (4.12).

Remark V.1 Notice that for $\mu<0$ (or $\left.\rho<\rho_{c}(\beta)\right)$, the space-averaged reduced density matrix (5.3) remains consistent with the free nonrandom case. Indeed, when $\lambda \downarrow 0$, then the second integral in (5.6) tends, by (3.11), to zero, whereas the first integral has, by (4.4), the limit:

$$
\lim _{\lambda \downarrow 0} \tilde{\rho}(x, y ; \beta, \mu)=\rho(x, y ; \beta, \mu),
$$

where

$$
\begin{aligned}
\rho(x, y ; \beta, \mu) & =\frac{1}{\pi} \int_{0}^{\infty} \frac{d E}{\sqrt{2 E}} \frac{1}{e^{\beta(E-\mu)}-1} \\
& \times \cos (\sqrt{2 E}(x-y))
\end{aligned}
$$

coincides with two-point correlation function of the free $P B G$ [13]. This equivalence is valid only when there is no condensation, since by (4.4) and (4.7) one has that $\lim _{\lambda \downarrow 0} \rho_{c}(\beta)=\infty$.

\section{COMMENTS AND DISCUSSION}

\section{A. Critical density}

We start by a remark concerning modifications of the Luttinger-Sy model properties, and in particular of the value of the critical density, when one passes from an infinite to a finite amplitude $a<\infty$ of the $\delta$-potential (2.6).

Recall that operators $\left\{h_{a}^{\omega}\right\}_{a>0}$ correspond to a monotonically increasing family of quadratic forms with $h_{a=+\infty}^{\omega}=h_{D}^{\omega}$, see (2.8). Then by definition of the integrated density of states (3.10), (3.11) and by the minimax principle for $h_{D}^{\omega}$ and $h_{a}^{\omega}$, one gets

$$
\begin{aligned}
\mathcal{N}_{\lambda}(E) & \equiv \mathcal{N}_{\lambda, a=+\infty}(E)<\mathcal{N}_{\lambda, a}(E) \leq \mathcal{N}_{\lambda, a=0}(E) \\
& =\mathcal{N}_{\lambda=0, a}(E)=\frac{\sqrt{E}}{c}
\end{aligned}
$$

Notice that the integrated density of states for the free case $a=0$ coincides with that for the zero impurity density (3.15), $\lambda=0$. From (6.1) one gets the corresponding inequalities for critical densities (4.7) indicating the enhancement of the BEC that was remarked already by Kac and Luttiger [8,9].

More refined arguments (see e.g. [20], Ch.III, 6B) give for $E<\pi^{2} a^{2} / 32$, for the estimate, cf. (3.14):

$$
\mathcal{N}_{\lambda}(E)<\mathcal{N}_{\lambda, a}(E)<\lambda \sum_{s=1}^{\infty} e^{-s \lambda(c / \sqrt{E}-4 / a)}=: \mathcal{N}_{\lambda, a}^{*}(E)
$$

Then, the definition of the critical density (4.7) and (6.1) yield

$$
\rho_{c}(\beta, \lambda):=\lim _{\mu \uparrow 0} \int_{0}^{\infty} \frac{d \mathcal{N}_{\lambda}(E)}{e^{\beta(E-\mu)}-1}=\int_{0}^{\infty} d E \mathcal{N}_{\lambda}(E) \frac{\beta e^{\beta E}}{\left(e^{\beta E}-1\right)^{2}} \leq \int_{0}^{\infty} d E \mathcal{N}_{\lambda, a}(E) \frac{\beta e^{\beta E}}{\left(e^{\beta E}-1\right)^{2}}=: \rho_{c}(\beta, \lambda, a)
$$

with obvious limits: $\lim _{a \rightarrow 0} \rho_{c}(\beta, \lambda, a)=\infty$ and $\lim _{\lambda \rightarrow 0} \rho_{c}(\beta, \lambda, a)=\infty$, by (6.1).

The estimate $(6.2)$ yields the upper boundary on $\rho_{c}(\beta, \lambda, a)$ for the small $a$. Setting $\tilde{E}(a):=(\pi a / 8)^{2}$ by $(6.1)-(6.3)$ we get that

$$
\rho_{c}(\beta, \lambda, a) \leq \int_{0}^{\tilde{E}(a)} d E \mathcal{N}_{\lambda, a}^{*}(E) \frac{\beta e^{\beta E}}{\left(e^{\beta E}-1\right)^{2}}+\int_{\tilde{E}(a)}^{\infty} d E \frac{\sqrt{E}}{c} \frac{\beta e^{\beta E}}{\left(e^{\beta E}-1\right)^{2}}=: I(\beta, \lambda, a) .
$$

Then for fixed $\lambda>0$ and small $a>0$ we obtain the estimate:

$$
\rho_{c}(\beta, \lambda, a) \leq I(\beta, \lambda, a) \leq \frac{1}{\beta \lambda}\left(\frac{8}{\pi}\right)^{2} \frac{1}{4 e(\sqrt{2}-1)}+\frac{1}{a} \frac{16 \sqrt{2}}{\beta \pi^{2}} .
$$

Similarly, for $a>0$ and small $\lambda>0$ we obtain by (6.3) that

$$
\rho_{c}(\beta, \lambda, a) \leq \frac{1}{\lambda \beta} \int_{0}^{\tilde{E}(a)} \frac{d x}{x^{2}} \frac{e^{-c / \sqrt{x}}}{1-e^{-c / \sqrt{x}}}+\int_{\tilde{E}(a)}^{\infty} d E \frac{\sqrt{E}}{c} \frac{\beta e^{\beta E}}{\left(e^{\beta E}-1\right)^{2}} .
$$


Notice that the bounded critical density $\rho_{c}(\beta, \lambda, a)$ for $\lambda>0$ and $a>0$ is the key criterion of the existence of BEC in the one-dimensional system with integrated density of states $\mathcal{N}_{\lambda, a}(E)$, cf. (4.12). On the other hand the Bogoliubov-Hohenberg theorem says that there is no BEC in translation invariant boson systems if the dimension is less than $d=2$, see e.g. [3,4]. Therefore, the $\mathrm{BEC}$ in the Luttinger-Sy model is of a different nature than the case without a random impurity potential.

In fact, the randomness of the impurity potential is not indispensable for BEC in one-dimensional perfect Bosegas. To this end we construct nonrandom hierarchical models with impurity potential which manifest the BEC via a mechanism similar to that in the Luttinger-Sy model.

\section{B. Hierarchical model for BEC in one-dimensional nonrandom intervals}

We present here a nonrandom hierarchical onedimensional system, which manifests BEC and in a certain sense mimics the Luttinger-Sy model.

Type I BEC. Let $\Lambda:=(0, L)$ be a segment separated into $n$ impenetrable intervals of lengths $L_{j}, j=1, \ldots, n$ such that $\lambda=n / L<\infty$. For simplicity we take the hierarchy when all intervals, except the first (largest) one, are identical:

$$
L_{1}=\frac{\ln (\lambda L)}{\lambda} \quad \text { and } \quad L_{j \neq 1}=\tilde{L}_{n}=\frac{L-L_{1}}{n-1} .
$$

Then one gets

$$
\lim _{L \rightarrow \infty} L_{1}=+\infty \quad \text { and } \quad \lim _{L \rightarrow \infty} \tilde{L}_{n}=\frac{1}{\lambda} .
$$

This non-random system presents an obvious analogue of the Luttinger-Sy model. Here again, the quantum states are defined in independent intervals and they have energies

$$
E_{j, s}=\frac{c^{2} s^{2}}{L_{j}^{2}}, j=1, \ldots, n, s=1,2, \ldots,
$$

with $c^{2}=\pi^{2} / 2$. The spectrum of the corresponding Schrödinger operator is discrete and bounded below by zero, cf. (2.15), (2.17). Then the chemical potential is $\mu<0$ and the PBG particle density in $\Lambda$ has the same expression as in (4.5)

$$
\rho_{L}(\beta, \mu)=\frac{1}{L} \sum_{j=1}^{n} \sum_{s=1}^{\infty} \frac{1}{e^{\beta\left(E_{j, s}-\mu\right)}-1}, \beta>0, \mu \leq 0 .
$$

By virtue of the hierarchical structure of intervals we can separate the expression for density into two parts:

$$
\begin{aligned}
\rho_{L}(\beta, \mu) & =\frac{1}{L} \sum_{s=1}^{\infty} \frac{1}{e^{\beta\left(c^{2} s^{2} / L_{1}^{2}-\mu\right)}-1} \\
& +\frac{n-1}{L} \sum_{s=1}^{\infty} \frac{1}{e^{\beta\left(c^{2} s^{2} / \tilde{L}_{n}^{2}-\mu\right)}-1} .
\end{aligned}
$$

Since $L_{1}=O(\ln (\lambda L))$, the first sum in (6.7) converges, when $L \rightarrow \infty$, to zero for all $\mu \leq 0$, i. e. we obtain

$$
\rho(\beta, \mu)=\lim _{L \rightarrow \infty} \rho_{L}(\beta, \mu)=\lambda \sum_{s=1}^{\infty} \frac{1}{e^{\beta\left((c s / \lambda)^{2}-\mu\right)}-1} .
$$

As a consequence, the critical density for this system is finite:

$$
\begin{aligned}
\rho_{c}(\beta): & =\sup _{\mu \leq 0} \rho(\beta, \mu)=\rho(\beta, 0) \\
& =\lambda \sum_{s=1}^{\infty} \frac{1}{e^{\beta(c s / \lambda)^{2}}-1}<\infty,
\end{aligned}
$$

and we have BEC condensation, when $\rho>\rho_{c}(\beta)$.

This condensation is of type $I$, since the difference between the ground-state energy and the energy of the first excited state (which are both localized in the biggest interval $\left.L_{1}\right)$ is of the order $O\left(L_{1}^{-2}\right)=O\left((\ln (\lambda L))^{-2}\right)$, see e. g. [1], or [25]. In this case the solution $\mu_{L}(\beta, \rho)$ of the equation

$$
\begin{aligned}
\rho & =\frac{1}{L} \frac{1}{e^{\beta\left(c^{2} / L_{1}^{2}-\mu\right)}-1} \\
& +\frac{1}{L} \sum_{s>1} \frac{1}{e^{\beta\left(\left(c s / L_{1}\right)^{2}-\mu\right)}-1} \\
& +\frac{\nu-1}{L} \sum_{s=1}^{\infty} \frac{1}{e^{\beta\left(\left(c s / \tilde{L}_{n}\right)^{2}-\mu\right)}-1},
\end{aligned}
$$

for $\rho>\rho_{c}(\beta)$ and large $L$ has asymptotics

$$
\mu_{L}(\beta, \rho)=E_{1,1}-\frac{1}{\beta \rho_{0}(\beta, \rho) L}+O\left(1 / L^{2}\right),
$$

see (6.6). Inserting (6.11) into (6.10) we obtain in the limit

$$
\begin{aligned}
\rho & =\lim _{L \rightarrow \infty} \frac{1}{L} \frac{1}{e^{\beta\left(c^{2} / L_{1}^{2}-\mu_{L}(\beta, \rho)\right)}-1}+\lambda \sum_{s=1}^{\infty} \frac{1}{e^{\beta(c s / \lambda)^{2}}-1} \\
& =\rho_{0}(\beta, \rho)+\rho_{c}(\beta)
\end{aligned}
$$

where by (6.11) the condensate density is

$$
\rho_{0}(\beta, \rho)=\lim _{L \rightarrow \infty} \frac{1}{L} \frac{1}{e^{\beta\left(c^{2} / L_{1}^{2}-\mu_{L}(\beta, \rho)\right)}-1} .
$$

Therefore, this one-dimensional hierarchical model shows type I BEC localized in the (logarithmically) large, but not macroscopic, domain corresponding to the groundstate wave function. Generalizations to another hierarchy of intervals with one largest interval trapping BEC are obvious.

For example, it is easy to generalize the above observation to type $I$ BEC localized in a finite number of $M$ identical (logarithmically) large intervals: 


$$
L_{1}=\ldots=L_{M}=\frac{\ln (\lambda L)}{\lambda}
$$

and

$$
L_{j}=\tilde{L}_{n}=\frac{L-M L_{1}}{n-M}, M<j \leq n,
$$

cf. (6.4). Then similar to the case $M=1$ (6.7) one gets

$$
\begin{aligned}
\rho_{L}(\beta, \mu) & =\frac{1}{L} \sum_{j=1}^{M} \sum_{s=1}^{\infty} \frac{1}{e^{\beta\left(c^{2} s^{2} / L_{j}^{2}-\mu\right)}-1} \\
& +\frac{n-M}{L} \sum_{s=1}^{\infty} \frac{1}{e^{\beta\left(c^{2} s^{2} / \tilde{L}_{n}^{2}-\mu\right)}-1} .
\end{aligned}
$$

which implies through verbatim that for $M>1$ the critical density $(6.9)$ rests the same. If $\rho>\rho_{c}(\beta)$, the equation $\rho=\rho_{L}(\beta, \mu)$ (6.13) yields for asymptotics of the solution $\mu_{L}(\beta, \rho)$ an expression similar to $(6.11)$ for $M=1$ :

$$
\begin{aligned}
& \mu_{L}(\beta, \rho)=E_{j, 1}-\frac{M}{\beta \rho_{0}(\beta, \rho) L}+O\left(1 / L^{2}\right), \\
& 1 \leq j \leq M .
\end{aligned}
$$

Here $E_{1,1}=\ldots=E_{M, 1}$ by (6.6) and (6.12). Then taking into account $(6.6)$ and $(6.12),(6.14)$ we obtain by (6.13)

$$
\begin{aligned}
\rho & =\lim _{L \rightarrow \infty} \frac{1}{L} \sum_{j=1}^{M} \frac{1}{e^{\beta\left(c^{2} / L_{j}^{2}-\mu_{L}(\beta, \rho)\right)}-1} \\
& +\lambda \sum_{s=1}^{\infty} \frac{1}{e^{\beta(c s / \lambda)^{2}}-1}=\rho_{0}(\beta, \rho)+\rho_{c}(\beta),
\end{aligned}
$$

where the condensate density is equally shared among the first $M$ intervals:

$$
\rho_{0}(\beta, \rho)=\lim _{L \rightarrow \infty} \frac{M}{L} \frac{1}{e^{\beta\left(c^{2} / L_{1}^{2}-\mu_{L}(\beta, \rho)\right)}-1} .
$$

Type II BEC. To obtain type II BEC in one interval we take, instead of (6.4):

$$
L_{1}:=\sqrt{L / \lambda} \quad \text { and } \quad L_{j \neq 1}=\tilde{L}_{n}=\frac{L-L_{1}}{n-1} .
$$

Then (6.7) gets the form

$$
\begin{aligned}
\rho_{L}(\beta, \mu) & =\frac{1}{L} \sum_{s=1}^{\infty} \frac{1}{e^{\beta\left(\lambda c^{2} s^{2} / L-\mu\right)}-1} \\
& +\frac{n-1}{L} \sum_{s=1}^{\infty} \frac{1}{e^{\beta\left(c^{2} s^{2} / \tilde{L}_{n}^{2}-\mu\right)}-1} .
\end{aligned}
$$

Since for $\mu \leq 0$ the first sum in (6.16) converges to zero, when $L \rightarrow \infty$, we obtain for $\rho(\beta, \mu)=\lim _{L \rightarrow \infty} \rho_{L}(\beta, \mu)$ and $\rho_{c}(\beta)$ the same expressions as in (6.8) and (6.9).

Now, if $\rho>\rho_{c}(\beta)$, the solution $\mu_{L}(\beta, \rho)$ of equation $\rho=\rho_{L}(\beta, \mu)$ has asymptotics defined by $(6.16)$ :

$$
\begin{aligned}
\rho & =\lim _{L \rightarrow \infty} \frac{1}{L} \sum_{s=1}^{\infty} \frac{1}{e^{\beta\left(\lambda c^{2} s^{2} / L-\mu_{L}(\beta, \rho)\right)}-1} \\
& +\lambda \sum_{s=1}^{\infty} \frac{1}{e^{\beta(c s / \lambda)^{2}}-1}=\rho_{0}(\beta, \rho)+\rho_{c}(\beta) .
\end{aligned}
$$

As in (6.11) this implies

$$
\mu_{L}(\beta, \rho)=E_{1,1}-\frac{A(\beta, \rho)}{\beta L}+O\left(1 / L^{2}\right),
$$

see (6.6), where by (6.17) the coefficient $A(\beta, \rho) \geq 0$ satisfies the equation

$$
\rho=\sum_{s=1}^{\infty} \frac{1}{\beta \lambda c^{2}\left(s^{2}-1\right)+A}+\rho_{c}(\beta) .
$$

Hence, for $\rho>\rho_{c}(\beta)$ the BEC

$$
\rho_{0}(\beta, \rho)=\sum_{s=1}^{\infty} \frac{1}{\beta \lambda c^{2}\left(s^{2}-1\right)+A(\beta, \rho)}
$$

is split between infinitely many states in the largest interval $L_{1}$, i. e. this is the van den Berg-Lewis-Pulé condensation [1] of the type II.

Type III BEC. Now we show that (unusual) spatially fragmented type III BEC is possible in our hierarchical model. To split BEC between infinitely many states in different intervals, let volume $\Lambda$ be occupied by $[\ln (n+1)]$ identical (logarithmically) large intervals:

$$
\begin{aligned}
& L_{j}=\frac{\ln (\lambda L)}{\lambda}, \quad 1 \leq j \leq[\ln (n+1)]=: M_{n} \\
& \text { and } \\
& L_{j>M_{n}}=\tilde{L}_{n}:=\frac{L-L_{1} M_{n}}{n-M_{n}},
\end{aligned}
$$

for $M_{n}<j \leq n$, of small intervals, cf. (6.12). Then (similar to $(6.13))$ we get for the particle density

$$
\begin{aligned}
\rho_{L}(\beta, \mu) & =\frac{1}{L} \sum_{j=1}^{M_{n}} \sum_{s=1}^{\infty} \frac{1}{e^{\beta\left(c^{2} s^{2} / L_{j}^{2}-\mu\right)}-1} \\
& +\frac{n-M_{n}}{L} \sum_{s=1}^{\infty} \frac{1}{e^{\beta\left(c^{2} s^{2} / \tilde{L}_{n}^{2}-\mu\right)}-1}
\end{aligned}
$$

Since by $(6.20)$ we have $\lim _{L \rightarrow \infty} \tilde{L}_{n}=$ $\lim _{L \rightarrow \infty}\left(n-M_{n}\right) / L=\lambda$ the critical density (6.9) remains the same. If $\rho>\rho_{c}(\beta)$, the equation $\rho=\rho_{L}(\beta, \mu)$ (6.21) yields for asymptotics of the solution $\mu_{L}(\beta, \rho)$ :

$$
\begin{aligned}
& \mu_{L}(\beta, \rho)=E_{j, 1}-\frac{M_{n}}{\beta \rho_{0}(\beta, \rho) L}+O\left(1 / L^{2}\right), \\
& 1 \leq j \leq M_{n} .
\end{aligned}
$$

Then we obtain for the particle density in large intervals 


$$
\begin{aligned}
& \lim _{L \rightarrow \infty} \frac{1}{L} \sum_{j=1}^{M_{n}} \sum_{s=1}^{\infty} \frac{1}{e^{\beta\left(c^{2} s^{2} / L_{j}^{2}-\mu_{L}(\beta, \rho)\right)}-1}=\lim _{L \rightarrow \infty} \frac{M_{n}}{L} \frac{1}{e^{M_{n} /\left(\rho_{0}(\beta, \rho) L\right)-O\left(1 / L^{2}\right)}-1} \\
& +\lim _{L \rightarrow \infty} \frac{M_{n}}{L} \sum_{s=2}^{\infty} \frac{1}{e^{\beta\left((\lambda c s / \ln n)^{2}-\mu_{L}(\beta, \rho)\right)}-1}=\rho_{0}(\beta, \rho) .
\end{aligned}
$$

Limits (6.23) and (6.21), (6.22) imply that the condensate density $\rho_{0}(\beta, \rho)=\rho-\rho_{c}(\beta)$ is split between ground states of infinitely many logarithmic intervals in such a way that the condensate density in each interval is zero. This is a spatially fragmented type III BEC, which is different from the one corresponding to the simple dissimination over an infinite number of states and discussed by van den Berg, Lewis and Pulé in $[1,25]$.

\section{Statistics of large Poisson intervals}

By virtue of 6.2 to discriminate between possible types of BEC in the Luttinger-Sy model one has to study statistics of the size $\left\{L_{j}^{\omega}\right\}_{j}$ of intervals induced by Poisson distributed point impurities, see Proposition III.1.

In fact, the first attempt to elucidate this question is already contained in [18]. They gave some arguments in favour of a large finite $\Lambda$, the largest interval $I_{1}^{\omega}$ hasing a typical length of the logarithmic order:

$$
L_{1}^{\omega} \sim \lambda^{-1} \ln (\lambda L), L \rightarrow \infty
$$

Moreover, along the same line of reasoning they conclude that all other intervals $I_{j>1}^{\omega}$ are typically much smaller than $I_{1}^{\omega}$. Then neglecting the fluctuations in the length of $L_{1}^{\omega}$ they conclude that BEC has to follow the scenario we described in 6.2 as type I BEC, cf. (6.4) and (6.24).

To check these arguments and to bolster them by some rigorous reasonings we use Proposition III.1. First we note that the average length of the Poisson intervals is

$$
\mathbb{E}_{\sigma_{\lambda}}\left(L_{j_{s}}^{\omega}\right)=\lambda \int_{0}^{\infty} d L L e^{-\lambda L}=\lambda^{-1}
$$

i. e., the total average length of any sample of intervals $\left\{I_{j}^{\omega}\right\}_{j=1}^{k}$ is $k / \lambda$. We are interested in the density of the joint probability distributions generated by the events: $\left\{\omega \in \Omega: L_{j_{1}}^{\omega} \geq L_{j_{2}}^{\omega} \geq \ldots \geq L_{j_{k}}^{\omega}\right\}$. They have evidently the form

$$
d \sigma_{\lambda, k}^{>}\left(L_{j_{1}}, \ldots, L_{j_{k}}\right):=k ! \theta\left(L_{j_{1}}-L_{j_{2}}\right) \theta\left(L_{j_{2}}-L_{j_{3}}\right) \ldots \theta\left(L_{j_{k-1}}-L_{j_{k}}\right) d \sigma_{\lambda, k}\left(L_{j_{1}}, \ldots, L_{j_{k}}\right) .
$$

Then one gets for the joint probability density of the two largest intervals:

$$
d \sigma_{\lambda, k}^{>}\left(L_{j_{1}}, L_{j_{2}}\right) / d L_{j_{1}} d L_{j_{2}}=k(k-1) \lambda^{2} e^{-\lambda L_{j_{1}}} e^{-\lambda L_{j_{2}}}\left(1-e^{-\lambda L_{j_{2}}}\right)^{k-2} \theta\left(L_{j_{1}}-L_{j_{2}}\right) .
$$

Now, let $A(s, t)$ be defined for non-negative integers $s$ and $t$ by

$$
A(s, t):=\int_{0}^{\infty} d x \ln ^{s}(x) x^{t} e^{-x} .
$$

For $s \geq 1$ and $t \geq 1$ this function verifies the following identities:

$$
A(s, t)=t A(s, t-1)+s A(s-1, t-1), A(1, t)=t A(1, t-1)+\Gamma(t),
$$

where $\Gamma(t)$ stands for the Gamma-function:

$$
\Gamma(t)=\int_{0}^{\infty} y^{t-1} e^{-y} d y
$$

It is also convenient to introduce the Euler Beta-function in the form:

$$
B(\nu, t)=\int_{0}^{\infty} e^{-t y}\left(1-e^{-y}\right)^{\nu-1} d y=\frac{\Gamma(\nu) \Gamma(t)}{\Gamma(\nu+t)} .
$$

Then by (6.27) we get for the expectation value of the largest interval

$$
\begin{aligned}
& \mathbb{E}_{\sigma_{\lambda, k}^{>}}\left(L_{j_{1}}^{\omega}\right)=k \lambda \int_{0}^{\infty} L_{j_{1}} e^{-\lambda L_{j_{1}}}\left(1-e^{-\lambda L_{j_{1}}}\right)^{k-1} d L_{j_{1}} \\
& =-\frac{k}{\lambda}\left(\partial_{t} B(k, t)\right)_{t=1}=-\frac{k}{\lambda} \Gamma(k) \partial_{t}\left(\frac{\Gamma(t)}{\Gamma(t+k)}\right)_{t=1}=-\frac{k}{\lambda}(k-1) !\left(\frac{\partial_{t} \Gamma(t)}{k !}-\frac{\partial_{t} \Gamma(k+t)}{(k !)^{2}}\right)_{t=1} .
\end{aligned}
$$




\section{A. ZAGREBNOV}

Taking into account (6.28), (6.29) and the definition of the Gamma-function one gets from (6.30)

$$
\mathbb{E}_{\sigma_{\lambda, k}^{>}}\left(L_{j_{1}}^{\omega}\right)=\frac{A(1, k)}{k ! \lambda}-\frac{A(1,0)}{\lambda}=\frac{1}{\lambda} \sum_{s=1}^{k} \frac{1}{s} .
$$

Similarly, we obtain the expectation for the next interval

$$
\mathbb{E}_{\sigma_{\lambda, k}^{>}}\left(L_{j_{2}}^{\omega}\right)=\frac{A(1, k)}{k ! \lambda}-\frac{A(1,1)}{\lambda}=\frac{1}{\lambda} \sum_{s=2}^{k} \frac{1}{s} .
$$

By virtue of (6.31) and (6.32) the mean difference $\mathbb{E}_{\sigma_{\lambda, k}^{>}}\left(L_{j_{1}}^{\omega}-L_{j_{2}}^{\omega}\right)=1 / \lambda$ is independent of the number $k$ of intervals in the sample, whereas they have, for large $k$, the logarithmic size (cf. (6.24)):

$$
\mathbb{E}_{\sigma_{\lambda, k}^{>}}\left(L_{j_{1,2}}^{\omega}\right)=\frac{1}{\lambda} \ln (k)+\frac{1}{\lambda} P_{1,2}+O(1 / k),
$$

with respect to the total average sample length $k / \lambda$, here $P_{1}=\mathbf{C}:=0.577 \ldots$, is the Euler constant, and $P_{2}=\mathbf{C}-1$. Using (6.26) and (6.28), (6.29) we find that the variance of the difference between two largest intervals in the sample is also $k$-independent and has the form:

$$
\operatorname{Var}_{\sigma_{\lambda, k}^{>}}\left(L_{j_{1}}^{\omega}-L_{j_{2}}^{\omega}\right)=\frac{1}{\lambda^{2}}
$$

Moreover, by the joint probability distribution (6.27) we obtain for any $\delta>0$ that probability

$$
\mathbb{P}\left\{\omega: L_{j_{1}}^{\omega}-L_{j_{2}}^{\omega}>\delta\right\}=e^{-\lambda \delta}
$$

of the events $A_{k}(\delta)=\left\{\omega: L_{j_{1}}^{\omega}-L_{j_{2}}^{\omega}>\delta\right\}$, is independent of $k$ for an increasing sequence of samples $\left\{I_{j}^{\omega}\right\}_{j=1}^{k}$, when $k \rightarrow \infty$.

By 6.2 and (6.33), (6.34) we see that types $I I$ or $I I I$ BEC are impossible in the one largest logarithmic "box", since that total average length of the sample is $k / \lambda$. To exclude types I, II, III condensations via a space fragmentation between, e.g., two "boxes", we have to estimate the probability of the events corresponding to the state-energy spacings between two largest intervals. By 6.2 (see (6.14), (6.18), (6.22) and [13]) this spacing should be larger than the inverse of the total sample length, which is $(k / \lambda)^{-1}$. To this end it is sufficient to estimate the probability of the event $S_{k}(a>0, \gamma>0)$ corresponding to the spacing between ground states:

$$
\begin{aligned}
\mathbb{P}\left\{S_{k}(a, \gamma)\right\}: & =\mathbb{P}\left\{\omega: E_{s=1}\left(L_{j_{2}}^{\omega}(k)\right)\right. \\
& \left.-E_{s=1}\left(L_{j_{1}}^{\omega}(k)\right)>\frac{a}{k^{1-\gamma}}\right\} .
\end{aligned}
$$

Here we denote the energies in the sample $\left\{I_{j}^{\omega}\right\}_{j=1}^{k}$ by

$$
\begin{aligned}
& E_{s}\left(L_{j_{r}}^{\omega}(k)\right)=\frac{c^{2} s^{2}}{\left(L_{j_{r}}^{\omega}(k)\right)^{2}} \\
& r=1, \ldots, k, s=1,2, \ldots
\end{aligned}
$$

Notice that by (6.35) we obtain that there is a kind "repulsion" between energy levels in different intervals. Indeed,

$$
\begin{aligned}
& \mathbb{P}\left\{S_{k}(a, \gamma)\right\} \geq \mathbb{P}\left\{\omega: L_{j_{1}}^{\omega}(k)-L_{j_{2}}^{\omega}(k)>\frac{a}{2 c^{2} k^{1-\gamma}} L_{j_{1}}^{\omega}(k)\left(L_{j_{2}}^{\omega}(k)\right)^{2}\right\} \\
& =\int_{0}^{\infty} \int_{0}^{\infty} d \sigma_{\lambda, k}^{>}(x, y) \theta\left(x-y-\frac{a}{2 c^{2} k^{1-\gamma}} x y^{2}\right)=: p_{k}(a, \gamma)
\end{aligned}
$$

where $\lim _{k \rightarrow \infty} p_{k}(a, 0<\gamma<1)=1$ by explicit calculations in (6.38). The same argument is valid for other than ground states as well as for intervals $\left\{I_{j_{r}}^{\omega}\right\}_{r>2}^{k}$ instead of $I_{j_{2}}^{\omega}$. Therefore, in this limit with the probability 1 the spacing is too large for a fragmentation of the condensate between the largest and other intervals.

\section{The Kac-Luttinger conjecture}

The above arguments prove the Kac-Luttinger conjecture in the case of the one-dimensional random Poisson potential of point impurities: for PBG the BEC is of type $I$ and it is localized in one "largest box".
To make this statement more precise recall that BEC exists only in the thermodynamic limit, which we construct as an increasing family of samples of intervals $\left\{I_{j}^{\omega}\right\}_{j=1}^{k}$ induced by the point impurities on $\mathbb{R}$. Since these random variables are independent, we can choose an increasing sequence of independent samples with one largest interval and with the property that

$$
\lim _{k \rightarrow \infty} \sum_{r=1}^{k} \mathbb{P}\left\{S_{k}(a, \gamma)\right\}=\infty,
$$

see (6.38). Then by the Borel-Cantelli lemma [22]

$$
\mathbb{P}\left\{\varlimsup S_{k}(a, \gamma)\right\}=1,
$$

where the event 


$$
\varlimsup S_{k}(a, \gamma):=\bigcap_{k=1}^{\infty} \bigcup_{l=k} S_{l}(a, \gamma)
$$

means that infinitely many events $\left\{S_{k}(a, \gamma)\right\}_{k>1}$ take place. Together with $\mathbf{6 . 3}$ statement $(6.40)$ means that with probability 1 in the thermodynamic limit $\mathbb{R}$ the BEC is localized in a single "largest box", and this con- densation is of type I.

\section{Acknowledgements}

This paper is a part of the common project with O. Lenoble, J. Pulé and Th. Jaeck. I would like to thank them for useful discussions of different aspects of the problem considered here.
[1] M. van den Berg, J. T. Lewis, J. V. Pulé, Helv. Phys. Acta. 59, 1271 (1986).

[2] P. Billingsley, Convergence of probability measures (Wiley, New York, 1968).

[3] N. N. Bogoliubov, Lectures on Quantum Statistics: Quasi-Averagies. Vol. 2 (Gordon and Breach, N.Y., 1970).

[4] M. Bouziane, Ph. A. Martin, J. Math. Phys. 17, 1848 (1976).

[5] T. C. Dorlas, L. A. Pastur, V. A. Zagrebnov, J. Stat. Phys. 124, 1137 (2006).

[6] M. P. A. Fisher, P. B. Weichman, G. Grinstein, D. S. Fisher, Phys. Rev. B 40, 456 (1989).

[7] H. L. Frish, S. P. Lloyd, Phys. Rev. 120, 1175 (1960).

[8] M. Kac, J. M. Luttinger, J. Math. Phys. 14, 1626 (1973).

[9] M. Kac, J. M. Luttinger, J. Math. Phys. 15, 183 (1974).

[10] M. Kobayashi, M. Tsubota, Phys. Rev. B 66, 174516-1 (2002).

[11] S. Kotani, Publ. Res. Inst. Math. Sci. 12, 477 (1976).

[12] O. Lenoble, Fonctions de corrélation de systèmes quantiques aléatoires. $\mathrm{PhD}$, Université de la Méditerranée, 2003.

[13] O. Lenoble, L. A. Pastur, V. A. Zagrebnov, Comptes-
Rendus de L'Académie des Sciences (Paris), Physique 5, 129 (2004).

[14] H. Leschke, P. Müller, S. Warzel, Markov Processes and Related Fields 9, 729 (2003).

[15] J. T. Lewis, J. V. Pulé, Commun. Math. Phys. 36, 1 (1974)

[16] I. M. Lifshitz, S. A. Gredeskul, L. A. Pastur, Introduction to the theory of disordered systems (Wiley-Interscience, N.Y., 1989).

[17] J. M. Luttinger, H. K. Sy, Phys. Rev. A 7, 701 (1973).

[18] J. M. Luttinger, H. K. Sy, Phys. Rev. A 7, 712 (1973).

[19] L. A. Pastur, Comm. Math. Phys. 75, 179 (1980).

[20] L. A. Pastur, A. Figotin, Spectra of Random and AlmostPeriodic Operators (Springer-Verlag, Berlin, 1992).

[21] O. Penrose, L. Onsager, Phys. Rev. 104, 576 (1956).

[22] A. N. Shiryayev, Probability (Springer-Verlag, Berlin, 1984).

[23] D. Ueltschi, Progr. Probab. 51, 363 (2002).

[24] C. N. Yang, Rev. Mod. Phys. 34, 694 (1962).

[25] V. A. Zagrebnov, J.-B. Bru, Phys. Rep. 350, 291 (2001).

[26] R. M. Ziff, G. E. Uhlenbeck, M. Kac, Phys. Rep. 32, 169 (1977).

\title{
КОНДЕНСАЦІЯ БОЗЕ-АЙНШТАЙНА В НЕВПОРЯДКОВАНИХ СЕРЕДОВИЩАХ
}

\author{
В. А. Загрєбнов \\ Середземноморсъкий університет і Центр теоретичної фізики, \\ Люміні-Каз, 907, Марсель, F-13288, Седе, 09, Франиія
}

Ми вивчаємо конденсацію одновимірного ідеального бозонного газу, вміщеного у випадковий потенціял Пуассона, що відповідає одиночним точковим домішкам. У моделі виявлено бозе-айнштайнівську конденсацію muny I, локалізовану в одному "найбільшому" інтервалі логарифмічного розміру (гіпотеза КацаЛютінгера). Показано, що математичний механізм конденсації Бозе-Айнштайна в такій випадковій моделі ідентичний до конденсації в одновимірній невипадковій ієрархії моделі масштабованих інтервалів. 